\title{
LA POLÍTICA EXTERIOR DE ESPAÑA HACIA ÁFRICA SUBSAHARIANA A TRAVÉS DEL ANÁLISIS CRÍTICO DE LOS PLANES ÁFRICA
}

\author{
María Ángeles Alaminos ${ }^{1}$ \\ UNISCI / Universidad Complutense de Madrid (UCM)
}

\begin{abstract}
Resumen:
El aumento de la atención de España hacia África Subsahariana durante los últimos años se ha visto reflejado en la elaboración de sucesivos planes de acción dotados de objetivos e iniciativas hacia el continente, destacando la elaboración del "Plan África 2006-2008" y el actualmente en vigor "Plan África 2009-2012". No obstante y aunque se ha activado la agenda política española en África Subsahariana, no se ha conseguido mirar hacia África con un enfoque comprehensivo y un diálogo a todos los niveles que nos permita conocer las necesidades concretas para poder adecuar de forma más eficaz los objetivos en sectores específicos y las políticas adaptadas a las distintas regiones o países.
\end{abstract}

Palabras clave: Política exterior, España, África Subsahariana, Plan África.

Title in English: "Spain's Foreign Policy towards Sub-Saharian Africa seen through a Critical Assessment of the Africa Plans".

\begin{abstract}
:
The Spanish increased attention to sub-Saharan Africa in recent years has been reflected in the successive development of action plans with different initiatives and objectives for the continent. We would highlight the development of the "Africa Plan 2006-2008" and the current "Africa Plan 20092012". However, and in spite of the growing Spanish political agenda in Sub-Saharan Africa, it has not espoused a comprehensive approach to Africa nor has it provided an all levels dialogue. This dialogue at all levels would enable the Government to meet specific needs in order to more effectively align targets within specific sectors and would allow policies to be tailored to different regions or countries.
\end{abstract}

Keywords: Foreign Policy, Spain, Sub Saharan Africa, Africa Plan.

Copyright $\odot$ UNISCI, 2011.

Las opiniones expresadas en estos artículos son propias de sus autores, y no reflejan necesariamente la opinión de UNISCI. The views expressed in these articles are those of the authors, and do not necessarily reflect the views of UNISCI.

\footnotetext{
${ }^{1}$ María Ángeles Alaminos Hervás es becaria de investigación en la Universidad Complutense de Madrid e investigadora de UNISCI.

Dirección: Departamento de Estudios Internacionales, Facultad de Ciencias Políticas y Sociología, UCM, Campus de Somosaguas, 28223 Madrid, España. E-mail: ma_alaminos_h@hotmail.com.

http://dx.doi.org/10.5209/rev_UNIS.2011.v27.38149
} 


\section{Introducción: Del escaso interés tradicional por Africa, al establecimiento de una nueva relación}

En el nuevo milenio, la atención de España hacia África Subsahariana se ha visto incrementada y reflejada en la elaboración de sucesivos planes de acción dotados de objetivos e iniciativas hacia el continente, desde la aprobación del "Plan de Acción para África Subsahariana" del año 2001 hasta la elaboración del "Plan África 2006-2008" y el actualmente en vigor "Plan África 2009-2012".

Si bien "la presencia y las relaciones institucionales de España con el África Subsahariana han sido tradicionalmente escasas y, en algunos casos, meramente testimoniales", en el nuevo milenio se intenta establecer una nueva relación y compromiso con el continente. ${ }^{2}$ Sin embargo, esta mayor implicación, ¿constituye una "urgencia ética" de la comunidad internacional centrada en el establecimiento de "relaciones de igualdad y respeto, así como en el reconocimiento de la soberanía e idiosincrasia de cada uno de estos países" ${ }^{3}$ o responde a la, en ocasiones, errónea percepción de amenazas provenientes del continente y desafiantes de la seguridad internacional, como "la proliferación de los "Estados fallidos", la conflictividad endémica, el crecimiento incontrolado de la población, los movimientos migratorios, el tráfico de drogas, las situaciones de hambruna, las enfermedades de alto riesgo, principalmente el sida, la piratería y el terrorismo de carácter islamista"?. ${ }^{4} \mathrm{En}$ el presente texto trataremos de dar respuesta a estos interrogantes mediante el análisis crítico de los Planes África.

Si los objetivos generales, las prioridades y las principales líneas de acción de la política española hacia África se mantienen prácticamente estables en los Planes África 20062008 y 2009-2012, podemos observar algunas diferencias significativas en la formulación de las prioridades geográficas así como disonancias relevantes en la adecuación de los objetivos con las prioridades geográficas y en la divergencia con los respectivos Planes Directores de la Cooperación Española. Intentaremos hacer explícitos algunos elementos de la política exterior española para reflejar la distancia entre el discurso institucional y las posibilidades reales de implementación en el terreno.

La política emprendida por el Gobierno español pretende además estar en la misma línea de acción que la política de la Unión Europea (UE). Las relaciones UE-África se han incrementado notablemente en los últimos años, favoreciendo el diálogo político y reforzando la cooperación. Algunos documentos fundamentales han visto la luz desde la celebración en El Cairo de la primera Cumbre UE-África, en el año 2000. Tras idear una "Estrategia para África" en 2005, la UE y África han redefinido su asociación, pasando de una estrategia “para” África a una asociación estratégica “con” África en 2007.

La Estrategia Conjunta UE-África (JAES) ha tratado de establecer una nueva relación entre Europa y África adoptando un enfoque comprehensivo que considera a África en su unidad y totalidad, se asienta sobre las relaciones de la Unión Europea con la Unión Africana como interlocutor privilegiado en el continente, y se cimienta en la construcción de un diálogo político profundo y a todos los niveles. Analizaremos las aspiraciones de la política exterior

\footnotetext{
${ }^{2}$ Moratinos, Miguel Ángel : "El compromiso ético y político con África”, Real Instituto Elcano, Análisis (20 de diciembre de 2005), p.1, en http://www.realinstitutoelcano.org/analisis/868/868_Moratinos.pdf

${ }^{3}$ Ibid., p.1.

${ }^{4}$ Fuente Cobo, Ignacio: "Visión geopolítica de África: "Visión española del África Subsahariana: Seguridad y Defensa", en Centro Superior de Estudios de la Defensa Nacional Documentos de Seguridad y Defensa, vol. 1 (2006), p. 22, en http://www.ceseden.es/centro_documentacion/documentos/1.pdf.
} 
española en paralelo con las de la UE en su intento de establecer una política exterior estructural hacia el continente africano. Las nuevas aproximaciones hacia África pretenden que el desarrollo no sea el único centro de las relaciones (en el marco del Acuerdo de Cotonú, 2000), sino que se amplíen los asuntos de interés mutuo y la cooperación a nivel político, económico y de seguridad. Abordaremos estos asuntos intentando desglosar las asimetrías y limitaciones institucionales.

\section{El Plan África 2006-2008. La retórica de la lucha contra la pobreza vs la realidad de la acción exterior basada en los intereses políticos, económicos v estratégicos}

\subsection{La formulación del Plan África 2006-2008 en el contexto internacional de la transformación de la agenda de desarrollo hacia África Subsahariana}

El Plan África 2006-2008 vio la luz en un momento fundamental de transformación de la agenda internacional de desarrollo ${ }^{5}$ y un año después de la formulación de la Estrategia de la UE para África y del Plan Director de la Cooperación española 2005-2008, influenciado por la Declaración del Milenio (2000) y los Objetivos de Desarrollo del Milenio (ODM) acordados durante dicha cumbre. ${ }^{6}$

Por un lado, la política africana de la Unión Europea tiene su origen en el Tratado de Roma y en la evolución posterior de una serie de acuerdos, comenzando por la Convención de Yaundé (1963-75), seguida de las Convenciones de Lomé (1975-2000) hasta llegar, en el año 2000, a la firma del Acuerdo de Cotonú con los países de África, Caribe y Pacífico (ACP). En el ámbito político, tras la celebración de la I Cumbre UE-África en El Cairo en el año 2000, que inició un diálogo político más estructurado entre los dos continentes, las relaciones avanzaron lentamente debido en parte a que los procesos de integración en ambos continentes se aceleraron. En 2001 se creó la Nueva Asociación para el Desarrollo de África (NEPAD) y, en 2002, la Unión Africana (UA).

Si bien la Unión Europea (o previamente la Comunidad Europea) ha mantenido relaciones institucionalizadas con África desde el principio del proceso de integración europeo, no fue hasta 2005 con la formulación de una Estrategia para África cuando la Unión adoptó un enfoque comprehensivo que enmarcara sus relaciones con el continente africano como un todo. ${ }^{7}$ La Estrategia para África supone "la respuesta de la Unión Europea al doble reto que consiste en volver a situar a África en la vía del desarrollo sostenible y en alcanzar los Objetivos de Desarrollo del Milenio (ODM) de aquí a 2015"8. Para lograr el desarrollo

\footnotetext{
${ }^{5}$ Nos referimos entre otros documentos a la Nueva Agenda de la Ayuda (Declaración de París de 2005)

6 "La Declaración del Milenio y otros acuerdos derivados de las Cumbres de Naciones Unidas ratificadas por España son los principales referentes del Plan Director", Plan Director de la Cooperación Española 2005-2008, p.5, en http://www.maec.es/SiteCollectionDocuments/Cooperaci\%C3\%B3n\%20espa\%C3\%B1ola/Publicaciones/Plan Director_Esp.pdf.

${ }^{7}$ Keukeleire, Stephan y MacNaughtan, Jennifer (2008): The Foreign Policy of the European Union, Houndmills, Palgrave Macmillan, p.288.

${ }^{8}$ Comunicación de la Comisión al Consejo, el Parlamento Europeo y el Comité Económico Social Europeo: Estrategia de la UE para África :Hacia un pacto euroafricano para acelerar el desarrollo en África, COM (2005) 489 final, Bruselas, 12 de octubre de 2005, p.44, en http://eur-lex.europa.eu/LexUriServ/LexUriServ.do?uri=COM:2005:0489:FIN:ES:PDF.
} 
sostenible de África, la Unión Europea identifica las condiciones previas indispensables: la paz y la seguridad.

Por otro lado, el Plan Director de la Cooperación española 2005-2008 se planteó el refuerzo "notable" de su compromiso con África Subsahariana, la "atención a las necesidades especiales de África" y "un incremento del esfuerzo y la calidad de la ayuda en el África Subsahariana, sin que ello incida en una excesiva dispersión geográfica". 9 El Plan Director estableció tres categorías de áreas geográficas y países de actuación: prioritarios (que en el caso de África Subsahariana incluían Mozambique, Angola, Namibia, Senegal y Cabo Verde), con atención especial (R.D. Congo, Etiopía, Guinea Ecuatorial, Sudán y Guinea Bissau), y preferentes (Santo Tomé y Príncipe, y Sudáfrica).

A pesar del mencionado interés en África Subsahariana, éste sigue siendo marginal y el plan especifica que "la ayuda responderá a los objetivos de la política exterior del Estado" y por tanto África Subsahariana no será una prioridad para la cooperación española, siendo áreas geográficas de actuación preferente "los países de Iberoamérica, los países árabes del Norte de África y de Oriente Medio, y aquellos otros de menor desarrollo con los que España mantenga especiales vínculos de carácter histórico y cultural". ${ }^{10}$

\subsection{Obietivos y prioridades geográficas}

El Plan África 2006-2008 planteó siete objetivos generales ambiciosos de la política española en África: ${ }^{11}$

1. Participación española en el afianzamiento de la democracia, la paz y la seguridad en África

2. Contribución de España a la lucha contra la pobreza y a la agenda de desarrollo de África Subsahariana

3. Fomento de la cooperación para ordenar los flujos migratorios

4. Participación activa en la Estrategia de la Unión Europea para África

5. Promoción de los intercambios comerciales y de la inversión. Especial atención a las relaciones pesqueras y la seguridad energética

6. Fortalecimiento de la cooperación cultural y científica y realización de proyectos culturales al servicio de los objetivos del desarrollo

7. Refuerzo de la presencia política e institucional española en África

En cuanto a las prioridades geográficas, el Plan establece cuatro categorías distintas: los países de interés prioritario (Guinea Ecuatorial, Senegal, Malí y Nigeria en África Central y Occidental; Angola, Namibia, Sudáfrica y Mozambique en África Austral; Kenia y Etiopía en África Oriental; Mauritania), los países de interés específico (Cabo Verde, Camerún, Gabón,

\footnotetext{
${ }^{9}$ Plan Director de la Cooperación Española 2005-2008, pp. 5, 17 y 23

${ }^{10}$ Ibid., p. 72.

${ }^{11}$ Plan África 2006-2008, Ministerio de Asuntos Exteriores y de Cooperación, pp.30 y ss., en http://www.maec.es/SiteCollectionDocuments/Home/LIBROPLANAFRICA.pdf.
} 
Gambia, Ghana, Guinea-Bissau, Níger, República de Guinea, Santo Tomé y Príncipe, Seychelles y Tanzania), países de especial seguimiento (Chad, Costa de Marfil, República Democrática del Congo, Sudán y Zimbabue) y organizaciones regionales prioritarias (Unión Africana y la CEDEAO: Comunidad Económica de Estados de África Occidental). ${ }^{12}$

Lo primero que observamos es que la definición de estas cuatro categorías de países no se corresponde con la denominación en tres categorías del Plan Director de la Cooperación Española y además los países incluidos en dichas categorías son también diferentes.

Tampoco hay coincidencia con el enfoque de la Estrategia de la UE para África, que pretende adoptar un enfoque comprehensivo y tratar al continente como a un todo. A pesar de que uno de los objetivos generales es la participación activa en la Estrategia de la Unión Europea para África, el Plan español se centra únicamente en África Subsahariana.

\subsection{Avances limitados en el fortalecimiento de las relaciones con África Subsahariana}

Al margen de estas contradicciones institucionales y a la vista de la amplia selección geográfica, no sólo podemos percibir la potencial dificultad de implementar el Plan en tan vasta extensión territorial, sino también la predominancia de algunos de los objetivos e intereses securitarios y económicos sobre otros objetivos de la política exterior española como la lucha contra la pobreza. "A cualquier observador se le generarían muchas dudas sobre la importancia de la lucha contra la pobreza como criterio determinante. De hecho, parece ser que son los intereses económicos (especialmente pesqueros y energéticos) y su relevancia estratégica como país de tránsito y origen de la inmigración los que determinan la condición de país de interés prioritario o país de especial seguimiento". ${ }^{13}$

Aunque el Plan supuso un documento de referencia para la nueva aproximación del Gobierno español al África Subsahariana, las limitaciones presupuestarias del $\operatorname{Plan}^{14}$ y el "énfasis en el uso de facilidades financieras y recursos para la defensa de los intereses inversores y comerciales españoles (pesca y energía), el control migratorio y la lucha contra el terrorismo" $" 15$ hacen que se aleje de la agenda de desarrollo de África Subsahariana y se enmarque en una perspectiva de la seguridad internacional propia de la posguerra fría que conlleva la fusión de los conceptos de desarrollo y seguridad. En esta óptica, el continente africano se ve por un lado como la fuente de diversas amenazas: subdesarrollo, inestabilidad, fragilidad de los Estados. Por otro lado, como el campo de acción e intervención internacional motivados por la necesidad de reaccionar y dar respuesta a dichas amenazas.

\footnotetext{
${ }^{12}$ Plan África 2006-2008, pp. 41 y ss., en http://www.maec.es/SiteCollectionDocuments/Home/LIBROPLANAFRICA.pdf.

${ }^{13}$ Alberdi Bidaguren y Bidaurratzaga Aurre, Eduardo: "El Plan África 2006-2008: Cuestiones para el debate", FRIDE: Foro AOD (25 de septiembre de 2006), p. 6, en http://www.fride.org/publicacion/330/el-plan-africa2006-2008--cuestiones-para-el-debate.

120 millones de euros para 2008 no parece suficiente para abordar todos los retos enumerados.

${ }^{15}$ Alberdi Bidaguren y Bidaurratzaga Aurre, op. cit., p. 2.
} 


\section{El Plan África 2009-2012: mismos obietivos, mismos intereses}

\subsection{En movimiento hacia un enfoque más regional: el auge de África Occidental como región prioritaria}

"El continente africano se ha convertido en una prioridad estratégica y política de la acción exterior española", así comienza el Plan África 2009-2012, que pretende establecer una política exterior hacia África Subsahariana más coherente que la del plan precedente.

En paralelo, el III Plan Director de la Cooperación Española (2009-2012), siempre con la referencia de los ODM, pretende, enlazando "de forma armónica con el anterior" plan, ser el "el PD la calidad y eficacia de la Cooperación Española". ${ }^{16}$ El PD recoge los compromisos de la Agenda de Acción de Accra (2008) y el compromiso de la UE con la eficacia de la ayuda: el Consenso Europeo sobre Desarrollo y el Código de Conducta de la UE sobre Complementariedad y División del Trabajo. En este sentido, y debido a la ausencia de lazos históricos en el continente similares a los de otros Estados miembros de la UE, "España, en un marco de complementariedad, debería considerar las oportunidades que existen para

establecer asociaciones entre donantes - ya sea como socio silencioso o activo - en África

\section{Subsahariana". 17}

El PD define prioridades geográficas a nivel de país ${ }^{18}$ y a nivel regional. Debemos destacar, a nivel regional, la consolidación de África Occidental como "región prioritaria" para la Cooperación Española, y la puesta en marcha de "un programa regional de cooperación, dotado de estructura, con una vertiente multilateral (CEDEAO) y otra bilateral que centrará su atención en estados en situación de fragilidad o posconflicto”. Este enfoque centrado en África Occidental se ve reflejado también en el Plan África 2009-2012, y demuestra la continuidad de la influencia del nexo seguridad-desarrollo en la formulación de la política exterior española hacia África Subsahariana.

En la elaboración del Plan África actual se manifiesta asimismo la influencia del marco de relaciones de la UE con África bajo el prisma de la Estrategia Conjunta UE-África (JAES) y del enfoque holístico centrado en el triángulo buen gobierno-desarrollo-seguridad. La JAES pretende superar los anteriores marcos de relación y asociación entre la UE y el continente africano, estableciendo una verdadera asociación entre iguales enfatizando la importancia del diálogo político al más alto nivel y considerando al continente africano en su unidad y totalidad. Sin embargo, la aplicación práctica de la Estrategia se ha visto limitada por diversos

\footnotetext{
16 Plan Director de la Cooperación Española, p.3, en http://www.casafrica.es/casafrica/Inicio/PlanDirectorCooperacionEspanola09-12.pdf. ${ }^{17}$ Ibid., p. 188 .

${ }^{18}$ Se establecen tres categorías de asociación diversas de las del anterior PD. Por un lado la llamada "asociación amplia" que en el caso de África Subsahariana incluye Etiopía, Malí, Mozambique, Senegal, Cabo Verde y Níger. Por otro lado una "asociación focalizada" (Guinea Ecuatorial, Sudán, Guinea Bissau, Gambia, Angola, República Democrática del Congo y Guinea Conakry) y por último la categoría de "asociación con países de renta media para la consolidación de logros de desarrollo" (Namibia).
} 
factores de índole político, institucional y financiero que han derivado en una política incoherente hacia el continente. ${ }^{19}$

\subsection{Obietivos v prioridades estratégicas regionales}

El Plan África 2009-2012 establece, prácticamente sin diferencias, los mismos objetivos y prioridades que el Plan 2006-2008. ${ }^{20}$

Por otro lado, el Plan África 2009-2012 introduce, aunque de forma poco concreta, un enfoque regional en el marco de las prioridades geográficas. Se distingue la dimensión continental (mediante la intensificación de la relación con la Unión Africana), la dimensión regional (CEDEAO, IGAD (Inter-Governmental Authority on Development), SADC (Comunidad de Desarrollo del África del Sur) y la Comunidad Económica de Estados de África Central) y la atención por países (veinticinco países parte de las anteriormente mencionadas comunidades económicas regionales). ${ }^{21}$

Nuevamente encontramos, como en el caso del Plan anterior, un excesivamente extenso ámbito territorial que dificulta la aplicación de políticas concretas y adaptadas a las diferentes regiones, en lugar de "apostar decididamente por un mayor nivel de concentración geográfica

\footnotetext{
${ }^{19}$ Véase al respecto Alaminos Hervás, María Ángeles (2010): “Aproximación a las relaciones contemporáneas entre la Unión Europea y África: La estrategia conjunta UE-África en el contexto de la Presidencia española del Consejo de la UE", en La Política Exterior de Seguridad y Defensa Común de la Unión Europea II: Resultados de la Presidencia española, UNISCI Papers 36, Madrid, UNISCI, pp. 125-148.

${ }^{20}$ Los principales objetivos y prioridades enumerados en el Plan África 2006-2008 y 2009-2012 (marcaremos estos últimos con letra en cursiva para la comparación) incluyen, respectivamente:

1. Participación española en el afianzamiento de la democracia, la paz y la seguridad en África

Objetivo 1: Apoyo a los procesos de consolidación de la democracia y construcción de la paz y la seguridad en África

2. Contribución de España a la lucha contra la pobreza y a la agenda de desarrollo de África Subsahariana

Objetivo 2: Contribución a la lucha contra la pobreza en África

3. Fomento de la cooperación para ordenar los flujos migratorios

Objetivo 4: Consolidación de la asociación con África en materia migratoria

4. Participación activa en la Estrategia de la Unión Europea para África

Objetivo 5: Refuerzo de la relación España-África por la vía multilateral y Unión Europea

5. Promoción de los intercambios comerciales y de la inversión. Especial atención a las relaciones pesqueras y la seguridad energética

Objetivo 3: Promoción de las relaciones comerciales y de inversión entre España y África, y del desarrollo económico africano

6. Fortalecimiento de la cooperación cultural y científica y realización de proyectos culturales al servicio de los objetivos del desarrollo

7. Refuerzo de la presencia política e institucional española en África

Objetivo 6: Consolidación de la presencia política e institucional española en África. Casa África y otras formas de diplomacia

21 "En África Occidental: además de Mauritania, los países de la CEDEAO, en especial Senegal, Malí,

Gambia, Costa de Marfil, Níger, Nigeria, Guinea Bissau, Guinea, Ghana y Cabo Verde. En el Cuerno de África: los países de IGAD, y en especial, Etiopía, Kenia y Sudán. En África Central: los países de CEEAC, y en especial, Guinea Ecuatorial, Camerún, Gabón y Santo Tomé y Príncipe. En el Sur de África: los países de SADC, y en especial, Sudáfrica, Namibia, Mozambique, Angola, Zimbabue, Tanzania y República Democrática del Congo". Plan África 2009-2012, MAEC, p. 69, en http://www.maec.es/es/Home/Documents/PLAN\%20AFRICA\%202009-2012_web.pdf.
} 
y sectorial, y especialmente sin pasar antes por una dispersión de la acción exterior que dificulte el proceso de concentración en el medio plazo". ${ }^{22}$

De forma reincidente observamos también la elección de los países bajo criterios más estratégicos y económicos que orientados al desarrollo, el fin de la pobreza o la consolidación de la democracia. La política exterior española no se centra en la cooperación al desarrollo sino en otros intereses de índole securitario y económico, lo cual hace diverger el Plan África y el PD. Podemos entrever el énfasis en el control de los flujos migratorios en África Occidental, el criterio económico y comercial en el Sur de África y los vínculos históricos con Guinea Ecuatorial. En el Cuerno de África las relaciones con Etiopía y Kenia favorecen las acciones multilaterales y el compromiso con Sudán es difícil debido al complejo contexto político sudanés, aunque España mantiene presencia política y de la AECID en su Embajada en Jartum.

\subsection{La necesidad de una mavor simetría v coherencia}

El nexo y la fusión de los conceptos de seguridad y desarrollo son patentes en la agenda exterior de España en África Subsahariana. El Plan Director de la Cooperación Española y el Plan África 2009-2012 se muestran divergentes y contradictorios en función de los intereses de uno y otro.

Para adquirir una mayor coherencia entre el discurso del Gobierno y la implementación real de las políticas "se necesita una firme voluntad política que lleve, por ejemplo, a ligar los nuevos Marcos de Asociación País de la AECID con el resto de acuerdos comerciales y de explotación (energética, pesquera, de recursos minerales), de modo que se beneficien los países africanos". 23

La misma situación de incoherencia la encontramos en el caso de la JAES. Si bien la estrategia plantea como una de sus prioridades estratégicas el fortalecimiento de la integración regional $^{24}$, en la práctica "la negociación por parte de Europa de Acuerdos de Asociación Económica (AAE) con Estados africanos se viene realizando de modo que menoscaban los objetivos de integración regional de la estrategia conjunta." ${ }^{25}$ La firma de acuerdos bilaterales dificulta la integración regional y se muestra incoherente con los objetivos de la JAES, al limitar la coordinación con los acuerdos regionales.

Además, si España quiere centrar su interés y apoyar la integración regional de África Occidental, debe reflexionar sobre las modalidades concretas de ofrecer ayuda efectiva para fortalecer (tanto mediante actuaciones bilaterales como multilaterales) las capacidades institucionales que permitan un mayor desarrollo e integración de la región. Los criterios

\footnotetext{
${ }^{22}$ Olivié, Illiana y Oya, Carlos: "Plan África 2009-12: se necesita más coherencia y concentración”, Política Exterior, no130 (Julio/Agosto 2009), en http://www.politicaexterior.com/archives/5568.

23 Manrique, Manuel: "La política exterior española en África: momento de recalibrar el enfoque", FRIDE Policy Brief, no 46 (Noviembre 2010),p. 4, en http://www.fride.org/publicacion/821/spain\%27s-foreign-policyin-africa:-time-to-reassess-the-vision.

${ }^{24}$ La UE pretende la integración socio-económica y política del continente que resulte en:

"An accelerated integration process with the participation of all stakeholders, including those in the informal sector; Improved coherence and convergence of the integration processes between the AU Commission and the RECs; Enhanced African capacities to implement the integration agenda; Synergies between African integration processes and the EPAs, the Euro-Mediterranean Partnership, and bilateral trade agreements." The Africa-EU Strategic Partnership: A Joint Africa-EU Strategy, p. 42, en http://ec.europa.eu/development/icenter/repository/EAS2007_joint_strategy_en.pdf.

${ }^{25}$ Bello, Oladiran: "La asociación UE-África: Una encrucijada estratégica", FRIDE Policy Brief, no 37 (Mayo 2010), p.3.
} 
centrados en el control de los flujos migratorios y en el terrorismo internacional se muestran polémicos y pueden obstaculizar la colaboración con los socios africanos.

\section{Conclusiones}

En el momento actual de las relaciones entre España y África Subsahariana, centrándonos fundamentalmente en documentos básicos como son los Planes África, podemos concluir que no se ha establecido el nuevo tipo de relación o asociación con el continente que se pretendía. El discurso institucional y la formulación de la política exterior española hacia África Subsahariana no se han correspondido con el compromiso firme con la agenda de desarrollo africana.

En este sentido, la agenda política española hacia África Subsahariana, que aúna los conceptos de seguridad y desarrollo y propone políticas comprehensivas, se ha visto ensombrecida en esta perspectiva analítica por la distancia insalvable entre la retórica de las declaraciones y la implementación efectiva en el terreno. Ello ha traído consigo la falta de una estrategia política real y la desconexión entre la formulación de objetivos universalistas y la realidad de la implementación de las operaciones en el terreno. ${ }^{26}$

La comunidad internacional ha elaborado un enfoque holístico que aúna los conceptos de seguridad, desarrollo económico y buen gobierno: "the idea that drives this triangular approach is that development can only be achieved in a secure and democratic environment, conducive to long-term investments". ${ }^{27}$

Sin embargo, este enfoque holístico triangular se caracteriza por la vaguedad y la ausencia de un verdadero enfoque político y "the loss of a strategic framework rather than the creation of one". ${ }^{28}$ Como expone un informe del International Peace Institute (Academy) "there is continued disconnect between the policy rhetoric about integrated securitydevelopment approaches at the international level and policy realities at the sectoral level". ${ }^{29}$

En nuestro caso de análisis podemos concluir que la política exterior de España hacia África Subsahariana sigue condicionada por intereses políticos y estratégicos en lugar de mirar hacia África con un enfoque comprehensivo y un diálogo a todos los niveles que nos permita conocer las necesidades concretas para poder adecuar de forma más eficaz los objetivos en sectores específicos y las políticas adaptadas a las distintas regiones o países.

\footnotetext{
${ }^{26}$ Marriage, Zoe (2006): Not breaking the rules, not playing the game: International assistance to countries at war, London, Hurst y Chandler, David (2006): Empire in denial: The politics of state-building, London, Pluto.

${ }^{27}$ Bagoyoko, N. y Gibert, Marie (2009): “The linkage between security, governance and development: The European Union in Africa", The Journal of Development Studies, vol. 45, no. 5 (2009), 789-814, p. 789.

${ }^{28}$ Chandler, David: "The security-development nexus and the rise of "anti-foreign policy", Journal of International Relations and Development, vol. 10, n4 (2007), pp. 362-386, p. 364

${ }^{29}$ IPA (2006): "The Security-Development nexus: Research findings and policy implications", Program report, New York, International Peace Academy, p.3.
} 